\title{
Security of the Digital Home Based on Recognizing the Face
}

\author{
Jyothi $\mathbf{R}^{\mathbf{1}}$, Lakshmisha S Krishna ${ }^{2}$ \\ PG Student, VLSI Design \& Embedded Systems, Alpha College of Engineering, Bengaluru, India ${ }^{1}$ \\ Assistant Professor \& HOD, Dept of Electronics \& Communication Engg, Alpha College of Engg, Bengaluru, India ${ }^{2}$
}

\begin{abstract}
In modern daily life, Smart security system has become absolutely necessary. To prevent robbery in sensitive areas such as home, banking sector etc. with low consumption of power and reliable security device this system has been proposed. By recognizing the face, it will allow only authorized people to enter the premises. The viola jones method is used to detect the face of the person by using the 'OpenCV' technique and RANSAC (RANdom SAmple Consensus) is used for face recognition. By matching with the database image the door opens for the known person automatically. For the strange person, the image will sent to owner's android application through Google Cloud Messaging. Based on his command, the door will be opened or closed. This security system is developed by using Atmega 328P electronic development board operated on battery power supply, wireless internet connectivity by using laptop and data base .This proposed system is more reliable and more effective. Compared to the other systems, this system will consumes very low data and power.
\end{abstract}

Keywords: Face Recognition, Arduino IDE,Data base, Bluetooth module, LCD, Android phone, Relays

\section{INTRODUCTION}

Face recognition is an efficient method for accessing the door control system for a wide range of safety and security application. For smart home, security is a very important aspect. It is adopting in most of the countries. The important aspect of door security is to accurately identify the persons. Face recognition became the natural way of performing authentication between humans. After the fingerprint technology, it has become most popular in biometric authentication trait. Bluetooth wireless technology is used access control systems. This system provides security for accessing the door using facial recognition for home premises. The web camera will capture the image of the person who is in front of the door and compare with the database. If the image of the person does not match with the database, the system will send a message to the Android application through bluetooth module. The complete face recognition is done by pressing single push button. The face of the person is detected by using viola jones method. For recognition of the face, 'OpenCV' technique and RANSAC (RANdom SAmple Consensus) is used. For classifying the feature of images which is stored in the database and for captured test images, Euclidean distance technique is used.

\section{LITERATURE SURVEY}

Hteik Htar Lwin and et al. Have proposed a door lock access system which comprised mainly of three subsystems: namely face recognition, face detection and automatic door access control. Face recognition is implemented by using the Principal Component Analysis (PCA). The door will open automatically for the known person due to the command of the microcontroller. On the other hand, alarm will ring for the unknown person. Drawback of this system is that input images are taken through a web camera continuously until the 'stop camera' button is pressed [1]. Someone is required at that location to check unauthorized person's images or status of the system and take further necessary action. Personal computer (PC) is connected with the microcontroller, the whole system will not work if PC is crashed or NonFunction.

Sadeque Reza Khan and et al. Have proposed a system contains sensors to detect obstacle, touch, smoke, heat and sound. The complete system is controlled by a PIC microcontroller 16F76. It gathers information from the sensors, makes a decision and sends a message to a corresponding number by using a GSM modem. If it finds any interruption in its sensors like if the IR is interrupted then PIC will send a message to the home owner and another message to the Police Station. In the same way for fire interruption a message will be sent to the fire brigade and other to the home owner [2]. In this system require extra hardware components like GSM Modem and Sensors. Alerts are sent through only message or SMS. 
B. Udaya Kumar and et al. presents the implementation of a low cost wireless home security system using remote access and ZigBee protocol through internet [3]. A ZigBee based star network with two nodes had been established using Xbee radio, PIC, MBED and ARM7 microcontroller. The detection of the gas leakage detection, intruder motion and visual surveillance of the home were provided with the help of Passive Infrared Sensor (PIR), Camera (LS_Y201) and Gas sensor (GH-312) and problem here is multiple micro controllers are used, usage of ZigBee based network to communicate with the base station is limited to 100-150 meters long distance only. Base station is dependent only on the Ethernet for internet connectivity.

J. Shankar Kartik and et al. have proposed two systems. One is based on GSM technology and other one uses web camera to detect the intruder. The first security system uses a web camera, installed in house environment, which is operated by software which is installed on the PC and uses Internet for communication. The camera detects the motion of any intruder in front of the camera range. The software communicates to the intended user through Internet network and at the same time it gives the sound alert. The second security system is SMS or message based and uses GSM technology to send the message to the owner [9].

\section{HARDWARE AND SOFTWARE}

\section{Hardware}

Arduino is an open-source electronics platform based on easy-to-use hardware and software. Arduino boards are able to read inputs - light on a sensor, a finger on a button, or a Twitter message - and turn it into an output - activating a motor, turning on an LED, publishing something online. You can tell the board what to do by sending a set of instructions to the microcontroller on the board. To do so you use the Arduino programming language (based on Wiring), and the Arduino Software (IDE), based on Processing. Thanks to its simple and accessible user experience, Arduino has been used in thousands of different projects and applications. The Arduino software is easy-to-use for beginners, yet flexible enough for advanced users. It runs on Mac, Windows, and Linux. Teachers and students use it to build low cost scientific instruments, to prove chemistry and physics principles, or to get started with programming and robotics. Designers and architects build interactive prototypes, musicians and artists use it for installations and to experiment with new musical instruments.

Bluetooth module HC-05 is a Bluetooth device which used for wireless communication with Bluetooth enabled devices (like smartphone). It communicates with microcontrollers using serial communication (USART). To enable Bluetooth communication the Bluetooth module is used widely. This module is then interfaced with the 8051 microcontrollers by using the UART and the data is transmitted in the form of packets. The path for the data transmission and reception is formed by using the pins of Tx and Rx of HC 05 Bluetooth module. The HC-05 has two operating modes, one is the Data mode in which it can send data and receive data from other Bluetooth devices and the other is the AT Command mode where the default device settings can be changed. We can operate the device in either of these two modes by using the key pin .It is very easy to pair the HC-05 module with microcontrollers because it operates using the Serial Port Protocol (SPP). Simply power the module with $+5 \mathrm{~V}$ and connect the Rx pin of the module to the Tx of MCU and Tx pin of module to Rx of MCU.

LCD (Liquid Crystal Display) is a flat panel display, electronic visual display, or video display that uses the light modulating properties of liquid crystals. These crystals do not emit light directly. LCDs are available to display arbitrary images (as in a general-purpose computer display) or fixed images which can be displayed or hidden, such as preset words, digits, and 7-segment displays as in a digital clock. They use the same technology, except that arbitrary images are made up of a large number of small pixels, while other displays have larger elements. These are used in a wide range of applications, including computer monitors, televisions, instrument panels, aircraft cockpit displays, and signage. They are common in consumer devices such as video players, gaming devices, clocks, watches, and telephones, and have replaced cathode ray tube (CRT) displays in most applications. They are available in a wider range of screen sizes than CRT and plasma displays, and since they do not use phosphors, they do not suffer image burn-in. LCDs are, however, susceptible to image persistence. The LCD screen is more energy efficient and can be disposed of more safely than a CRT. Its low electrical power consumption enables it to be used in batterypowered electronic equipment. This is basically has an

electronically modulated optical device made up of any number of segments filled with liquid crystals and arrayed in front of a light source (backlight) or reflector to produce images in color or monochrome. Liquid crystals were first discovered in 1888. By 2008, worldwide sales of televisions with LCD screens exceeded annual sales of CRT units; the CRT became obsolete for most purposes.

Android is a software stack for mobile devices that includes an operating system, middleware and key applications. The Android-SDK provides the tools and APIs necessary to begin developing applications on the Android platform using the Java programming language. 
Android is a platform that includes open source operating system, middleware and key applications for use on mobile devices based on the kernel Linux 2.6; in accordance with the principles of platform software design, based on available driver hardware device. One level up, the libraries of the platform are found and the required virtual machine for converting and runtime applications. These are complemented by basic applications needed for the basic management unit for the initial communication and use of the device by the user. At the top of the platform architecture, lie the applications developed by software developers. Android's mobile operating system is based on the Linux kernel. Google and other members of the Open Handset Alliance collaborated on Android's development and release. The Android Open Source Project (AOSP) is tasked with the maintenance and further development of Android. The Android operating system is the world's best-selling Smartphone platform. The first truly open and comprehensive platform for mobile devices, all of the software to run a mobile phone but without the any obstacles that have hindered mobile innovation.

Relay is an electromagnetically operated switch. It consists of a coil which is obtained by wounding the wire and an electro-magnetic switch. Magnetic field is developed this acts as a temporary magnet. This phenomenon is used in working of the relay. When a current is passed through the wire which is wound in form of a coil an electro-magnetic field is developed this acts as a temporary magnet. This phenomenon is used in working of relay. Relays are used as DC Motor Drivers. Here 2 relays are used. One for Opening the door and other is for closing the door.

\section{Software}

Arduino IDE (Integrated Development Environment)It has a text editor for writing the code, message area to display the errors and warnings, a text console, a tool bar with buttons and few menu options. Arduino IDE help us to connect to the Arduino hardware so that we can upload the programs. The program is developed by using embedded $\mathrm{C}$. $\mathrm{C}$ is the most widely used programming language for embedded processors/controllers. AT Commands are used for control GSM modems to communicate with them and do the required functions. The set of commands consists of a series of short text strings. Commands are used for operations such as dialing, hanging up and sending messages. In the beginning, a set of commands are required for establish a connection between the mobile operator and GSM modem. In this project, we are calling the AT commands from the program for sending, receiving and deleting messages. All these operations will be carried out automatically when the program run without any manual intervention for sending AT commands.

Database It is an organized collection of data, generally stored and accessed electronically from a computer system. Where databases are complex they are often developed by using formal design and modeling techniques. Access to this data is usually provided by a "database management system" (DBMS) consisting of an integrated set of computer software that allows users to interact with one or more databases and provides access to all of the data which is contained in the database. Small businesses can use databases in a number of different ways. A database can help to organize information about your customers and clients. A database can contain information about your product inventory. A database can track sales, expenses and other financial information. The best-known RDBMS using SQL to create and query databases are IBM DB2, Oracle, Microsoft Access and MySQL.

\section{IMPLEMENTATION}

There are two parts in this section. The first is the implementation of face recognition and the second is implementation of sending the request using Bluetooth.

Implementation of face recognition: This proposed system gives an idea for face recognition and it is implemented by using OpenCV technique which is a popular computer vision library. Recognizing the face is an important application of the image processing which is used in many fields. In our present life it can be used in many applications. This is the best choice after biometrics and fingerprint technology. This can be used in banking and security areas where we need authentication so that right authorized person can use it. The complete face recognition is done by pressing single push button. The face of the person is detected by using viola jones method. For recognition of the face, 'OpenCV' technique and RANSAC (RANdom SAmple Consensus) is used. For classifying the feature of images which is stored in the database and for captured test images, Euclidean distance technique is used.

Implementation of sending request using Bluetooth: This system pairs the HC-05 module with microcontrollers because it operates using the Serial Port Protocol (SPP).This module communicates with the help of USART at 9600 baud rate hence it is easy to interface with any microcontroller that supports USART. We can also configure the default values of the module by using the command mode. After setting the serial communication the request is sent to the authorized person. 


\section{Block Diagram}

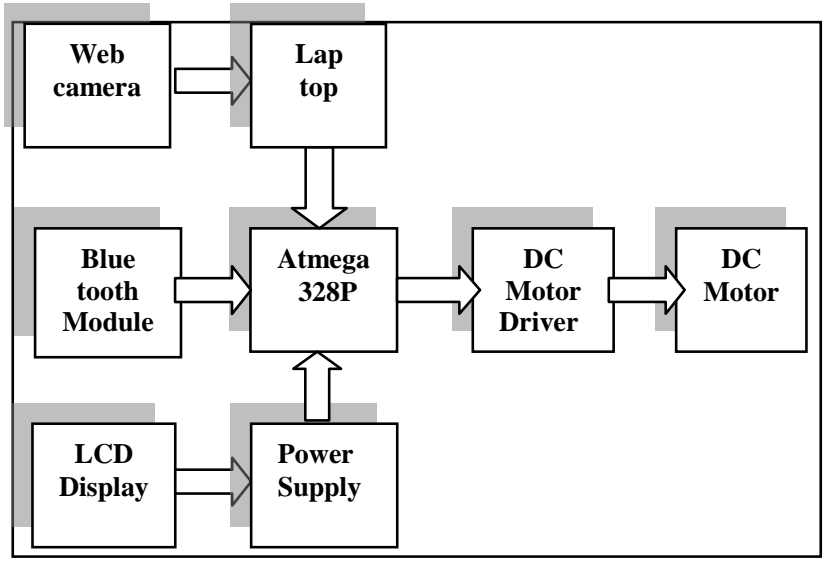

Fig.1 Block Diagram

This shows the block diagram of entire system. As shown above in block diagram, the system has Arduino board, DC Motor drivers, DC Motor, LCD Display, power supply and a bluetooth connector. The main control unit consists of ATmega 328P micro-Controller along with Arduino board. The battery power supply unit will be giving the supply to entire unit. It also has voltage regulator IC 7805.Bluetooth module provides serial communication to the Laptop.LCD for displaying the operation which is carried out. DC motor driver controls the operation of DC motor.

\section{Hardware Connections set up}

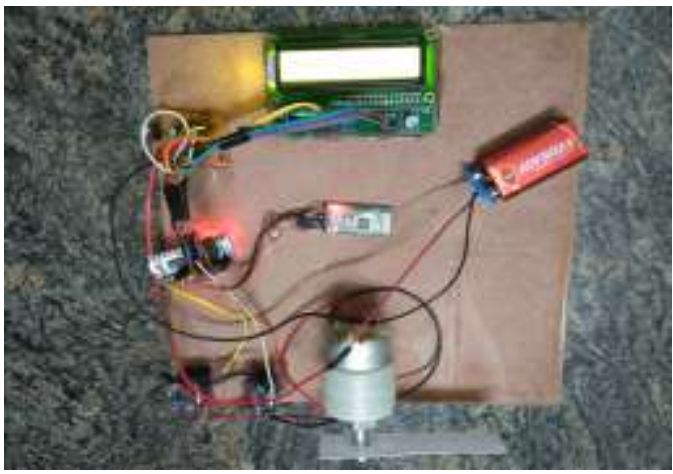

Fig.2: Hardware connection set up

The Hardware set up is the most important part in the project. The electronics components used in this are very simple and it includes connection of many hardware devices to the Arduino board. The following diagram shows the connection of required hardware components of this project like Arduino board, Relays, DC Motor, Bluetooth module, LCD and Power supply unit.

Software part is implemented by the following process

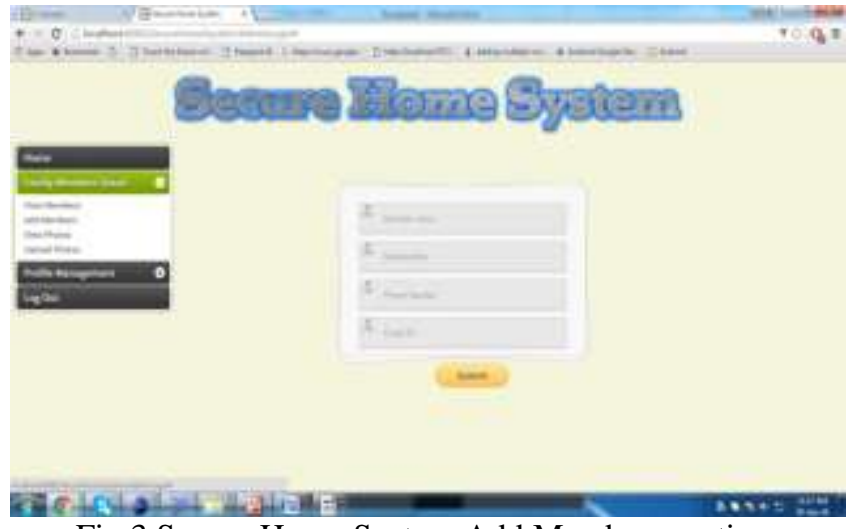

Fig.3 Secure Home System Add Members section 
The user can see the member details in the view members section. Admin can add new members in the system, admin can able to edit on existing member and delete the member in add members section.

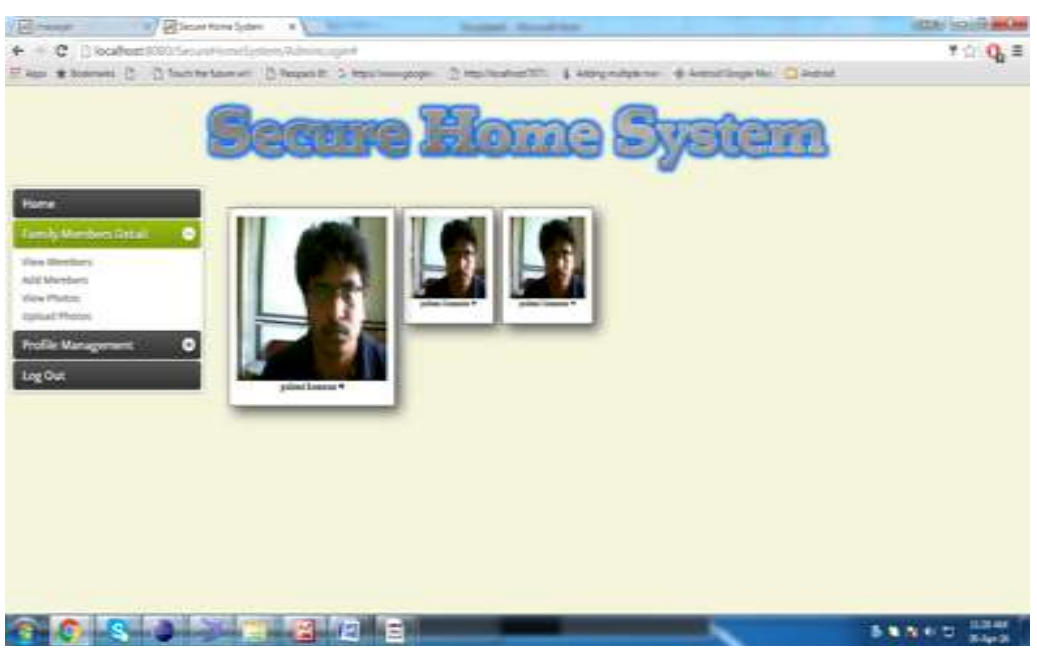

Fig. 4 Secure Home System View Photo section

The user has to upload his photo using web camera and he can see the uploaded image in the View Photos section.

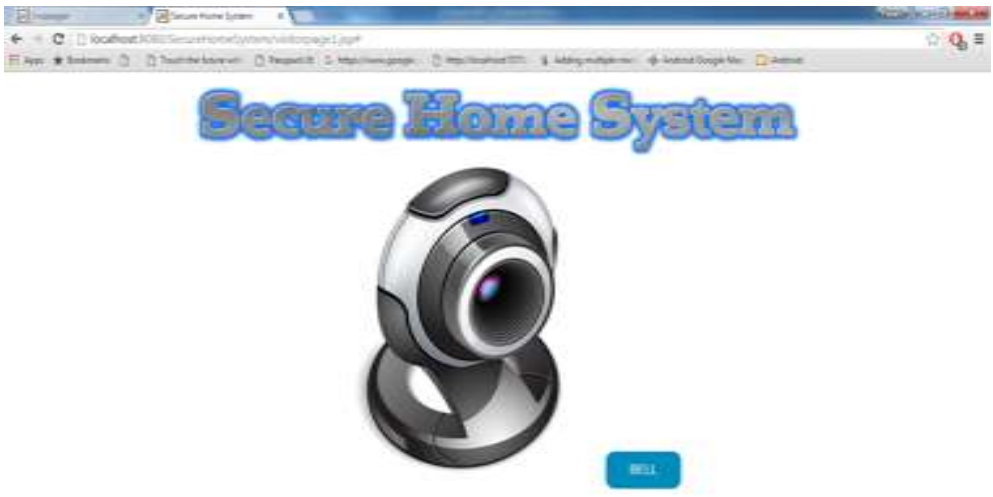

\section{Gilela}

Fig. 5 Secure Home System Visitor section

In this process visitor going to click on the bell that time cctv/ web camera will be getting started and capture the image.

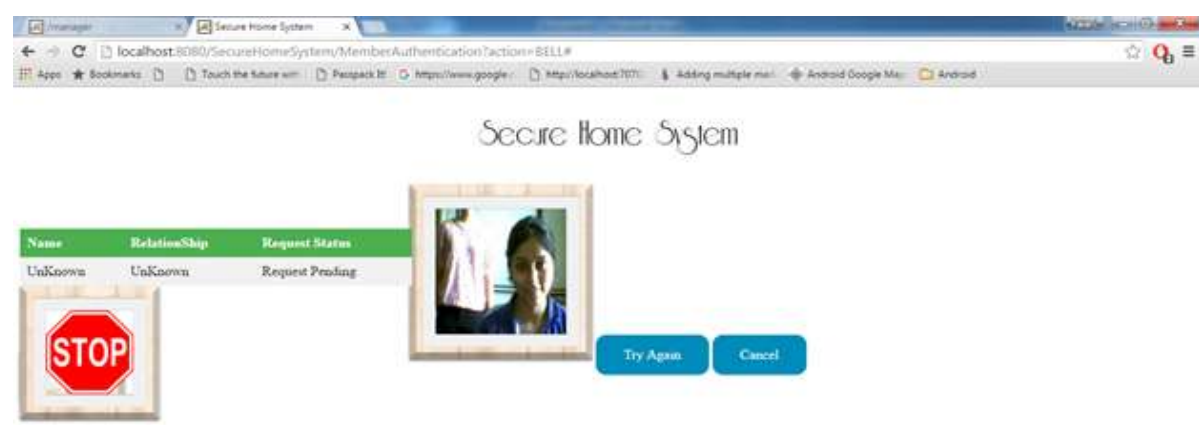


Vol. 8, Issue 4, April 2019

In this section admin can view visitor request with the photo and can able to allow request or reject, based on request status visitor will get the status message STOP/GO. If allowed door will be opened or else it be closed. If the image of the person does not match with the system database, the system will not allow and it will send the message to the Android phone through bluetooth.

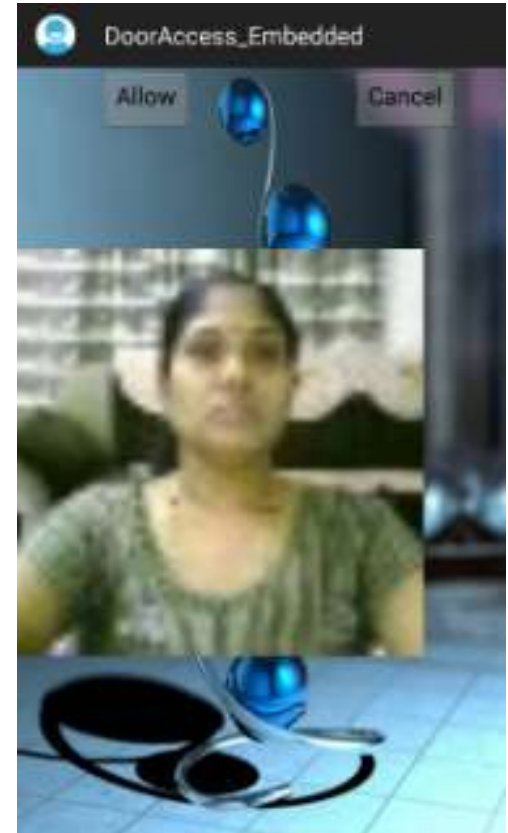

Fig. 7 screen shot of visitor request section

V. RESULTS

We have done the real time execution of this complete project. Results of project units are observed. The overall developed circuit looks as shown in the following figure:

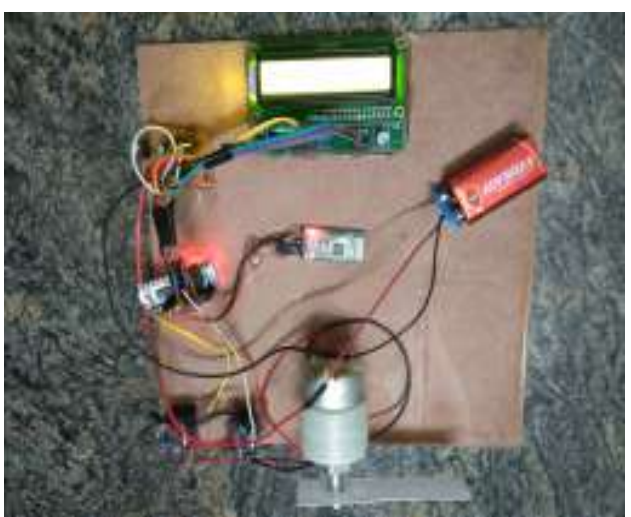

Fig. 8 LCD display showing result and bluetooth on

In this section authorized person can get the information of that person who is in front of the camera and he can decide whether to allow him or not.

\section{CONCLUSION}

In this project security of the home system by recognizing the face has been implemented. Face recognizing by verifying facial images and connecting to the Bluetooth paired device has been tested successfully. This system is wireless, reliable, robust and easily operated. It is a low cost security system. Wireless serial communication is achieved by using Bluetooth Connector through USART. The battery is connected to give the supply to the entire unit. Finally I conclude that all operations are tested successfully and results are documented. 


\title{
International Journal of Advanced Research in Computer and Communication Engineering
}

\author{
Vol. 8, Issue 4, April 2019
}

\section{REFERENCES}

[1]. Hteik Htar Lwin, Aung Soe Khaing, Hla Myo Tun, “Automatic Door Access System Using Face Recognition”, International Journal of Scientific \& Technology Research Volume 4, Issue 06, June 2015.

[2]. Sadeque Reza Khan, Ahmed Al Mansur, Alvir Kabir, Shahid Jaman, Nahian Chowdhury, "Design and Implementation of Low Cost Home Security System using GSM Network", International Journal of Scientific \& Engineering Research, Volume 3, Issue 3, March 2012.

[3]. B. Udaya Kumar, D. S. Murty, Ch. R. Phani Kumar, "Implementation of Low Cost Ethernet Based Home Security Using Wireless Sensor Network", Journal published at Algorithms Research, March 2013.

[4]. Jayashri Bangali, Arvind Shaligram, "Design and Implementation of Security Systems for Smart Home based on GSM technology", International Journal of Smart Home, Vol.7, No.6, August 2013.

[5]. V. Karri and J. S. Daniel Lim, "Method and Device to Communicate via SMS After a Security Intrusion", 1st International Confe-rence on Sensing Technology, Palmerston North, New Zealand, (2005) November 21- 23.

[6]. Mae .Y, Sasao .N, INNoue .K, Arai .T, "Person Detection by Mobile Manipulator for Monitoring", SICE 2003 Annual Conference, pages-28012806.

[7]. http://downloads.sourceforge.net/project/opencvlibrar y/opencv-2.4.zip

[8]. https://www.raspberrypi.org/products/model-b

[9]. J. Shankar Kartik, K. Ram Kumar and V.S. Srimadhavan3, "SMS Alert and Embedded Network Video Monitoring Terminal", International Journal of Security, Privacy and Trust Management ( IJSPTM), Volume 2, October 2013.

[10]. K. Yang, S. Ou, and H.H. Chen, "On Effective Offloading Services for Resource-Constrained Mobile Devices Run-ning Heavier Mobile Internet Applications," IEEE Comm. Magazine, vol. 46, no. 1, 2008, pp. 56-63.

[11]. C. Wang and Z. Li, "Parametric Analysis for Adaptive Com-putation Offloading," ACM SIGPLAN Notices, vol. 39, no. 6, 2004, pp. 119-130.

[12]. R. Wolski et al., “Using Bandwidth Data to Make Computa-tion Offloading Decisions," Proc. IEEE Int'l Symp. Parallel and Distributed Processing (IPDPS 08), 2008, pp. 1-8.

[13]. M. Newborn, “A Parallel Search Chess Program,” Proc. ACM Ann. Conf. Range of Computing: Mid-80’s Perspective, ACM Press, 1985, pp. 272-277.

[14]. J. Kincaid, “Google Privacy Blunder Shares Your Docs without Permission,” TechCrunch, 7 Mar. 2009; http:// techcrunch.com/2009/03/07/huge-google-privacy-blunder-sharesyour-docs-without-permission.

[15]. Muhammad Ali Mazidi, Janice Gillispie Mazidi, Rolin D. Mc Kinlay, The 8051 Microcontroller \& Embedded System, (Pearson Education Inc. 2 nd Edition, 2008) 